Domesticated

\section{and Then Some}

Jane Duran

University of California at Santa Barbara

Editors' Note: The following paper by

Professor Duran, and the commentary on it by Professor Varner, were presented at the Eastern Division meetings of the Society for the Study of Ethics and Animals, held in Atlanta, Georgia, December, 1989.

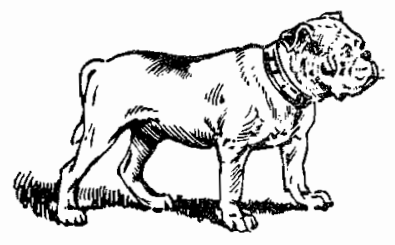

Questions about the rights of groups or kinds of animals, and their moral standing, have traditionally appeared in the literature as questions about species occurring in nature. " As Passmore has it, "Does it really matter that the moa no longer stalks the New Zealand plain?" In those few pieces where the status of domesticated species is discussed, however indirectly, the questions tend to center around commercial farming and arguments for and against vegetarianism, rather than the question of the status of domesticated vs. naturally occurring species as an issue in itself. ${ }^{2}$

In this paper I plan to address the question of the moral significance, from the standpoint of animal rights, of whether or not a species is domesticated. One hesitates to employ the term "species" here, because it turns out that what we are really talking about is not, in most cases of domesticated animals, the species itself but some subgrouping, usually created by humankind, frequently termed a breed. In other words, the question in its barest form is not whether horses or dogs have a moral standing different from that, say of the Bengal tiger. Horses and dogs existed before they were domesticated, and had they not come into extensive contact with people, would no doubt now be viewed by

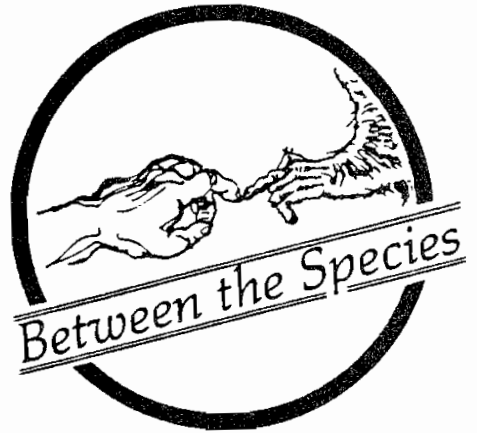

us in the same way that we view tigers. What we are really talking about, presumably, is the subgroups which humans have somewhat laboriously created from the original strains of domesticated animals. Dogs and horses, with their obvious gradations from St. Bernard to Pekingese and from Shire to Welsh pony, are only two cases in point. Many persons are surprised to discover that sheep, goats, pigs, ducks, geese, and fowl all exist in dozens of domesticated breeds, each one of which has recognizable characteristics, and each one of which typically has its own registry. Indeed, the creation of such breeds can itself become a viable financial enterprise; new registries for horses continually open up; and various characteristics are sought after, refined, and bred for, even when they first appear as only minor variations on the theme of an already existing breed. ${ }^{3}$

So, when we ask about the moral significance or lack there of of domesticated animals, we are inquiring, to reiterate, not about the distinction between the standing of an African elephant and a horse, but about the distinction, if any, between the status of an African elephant and a Shire horse. The crucial difference is, of course, that the Shire horse cannot reproduce itself in anything like its appropriate form without human assistance, since it is not a type of horse which occurs in nature. Were humans miraculously to vanish from the face of the earth tomorrow (not via nuclear warfare, since that, by hypothesis, would result in extreme

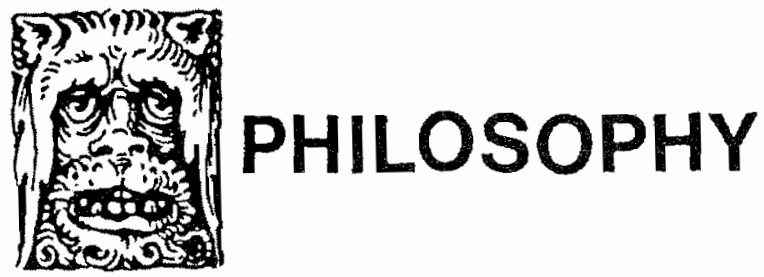


environmental contamination) elephants would continue indefinitely, but Shire horses would not.

Should we then be concerned about domesticated strains of creatures? Given that we have any concern at all for Passmore's moa, or for the ostrich, which is still here, should we have a similar concern for the Shire? I pick the Shire deliberately, since it is a breed of draft horse, and draft horses are not needed by humans in 1988 in the same way that they once were. In the past thirty years or so, the Shire has several times neared extinction, and has been rescued as a breed by a combination of British and American efforts. ${ }^{4}$ A great deal of time and money has been spent on the Shire, the Cleveland Bay, the Suffolk Punch, and even the Morgan ${ }^{5}$ (although it appears that the Morgan, for one, is long since home free); dog breeders are continually hearing about the plight of, for example, the Bemese Mountain Dog or the Redtick Hound.

On the face of it, it appears that domesticated breeds or subvarieties of species differ from their undomesticated counterparts in two important ways. The first and most obvious is that many breeds are ill-equipped for survival without human help. In some cases, particularly with certain dog breeds, muscular and skeletal changes through extensive and close inoreeding have contributed to the make-up of an animal who probably could not survive on its own. The dog's natural defenses have been virualily bred out. In oher cases, the animal could survive, but only in the sense that a given individual might be able to last out the course of its lifetime. Many breeds of horses are notoriously "shy" breeders (even outshying the Panda), and it is not clear that they would be able to reproduce without human intervention. The second major respect in which the human-created breeds differ from naturally occurring variations or species might be deemed to be a positive difference. It is, after all, these very animals who have contributed the most to the course of human history and culture. When horse breeders remind us of the role played by horses in the development of various human cultures, they are, after all, not speaking of zebras or Przewalski's horse. The horses they are speaking of were bred and raised by humans, and many of these horses are of the very same breeds now thought to be endangered.

On the other hand, one might want to note areas of similarity between the domestic breeds and nondomestic animals. Here one wants to say almost immediately that insofar as the characteristics having to do with putative moral significance are concerned, domesticated breeds are at least on a par with other animals. All domesticated creatures are, after all, sentient, just as the majority of non-domesticated creatures are, with the possible exception, as Singer notes, of molluscs and insects. ${ }^{6}$ Sentiency has widely been held to be the basis of the ascription of rights, or for utilitarian theories, the basis for the "taking into consideration" of animals and their well-being. Secondly, although it may very well be true that, given catastrophe and the absence of humans from the planet, Shire horses would revert to pre-Shire type, or even refuse to breed at all and die out within one generation, all animals, non-domestic and domestic, undergo subtle or not so subtle changes in mutable characteristics over a period of time. Thus, the reversion of most dogs to a mixed, indeterminate, pre-breed type without human intervention is simply a more dramatic illustration of the slower changes undergone, say, by the lion, over a period of time.

In "Darwin, Species and Morality," Rachels points out some respects in which differences between animals may not be as helpful in elucidating their moral status as one might have thought. What Rachels wants us to remember is that differences and distinctions are relevant only in specified contexts. Before developing this line of thought with regard to questions concerning animals, Rachels asks us to think in terms of differences between individual persons. Person A has higher grades and test scores than person $B$, but that only justifies giving A preferential treatment in situations that call for analysis along the lines of grades and test scoresnamely, academic situations. In another A-B duo, person A may be suffering from an infection and $B$ from a broken arm, but that only justifies making distinctions between them in a medical context. ${ }^{7}$ Rachels goes on to talk of the more standard sort of question in animal rights, i.e., whether or not I can justify my treatment of a chimpanzee, for example, by its presumed lesser capacity for rationality. ${ }^{8}$ But clearly, this type of distinction is relevant to the question we are discussing. The differences between domesticated and nondomesticated species are such that it is hard to see in what sorts of morally relevant contexts they would be applicable. The fact that a breed is ill-equipped for survival without human intervention, or even the happier fact that it contributed greatly to the development of human culture, does not immediately seem to be a morally relevant difference (particularly when we recall that the comparison here is between types of animals, and not between animals and humans). The 
fact that domesticated animals are also sentient and that their reversion to type is only a speeded-up version of general genetic change taking place in all living beings may be morally relevant, but those facts serve only to place domesticated animals more or less on a par with non-domesticated animals.

I want now to note another important point, also borrowed from Rachels' recent work, although with this particular point I will have to construct an analogy. Rachels' paper as a whole is concerned to argue for what he terms "Moral Individualism," meaning that we should regard animals, too, as individuals and not as typical members of their respective groups. In an interesting passage, Rachels notes that a hypothetical gifted chimpanzee who had learned to read and speak English might still be barred from the classroom by some well-meaning persons who contend that, in general, chimpanzees are not on a par with humans, and it is the group standards which count. As Rachels says, "This chimp is not permitted to do something which requires reading, despite the fact that he can read, because other chimps cannot. That seems not only unfair, but irrational." ${ }^{\circ 9}$

Now, we are not here concerned with Rachels' moral individualism, although in general I find his argument on that score convincing. But, constructing an analogy, I think the following may be said: domesticated animals differ from the non-domesticated in ways which are analogous to the differences between a very gifted chimpanzee and the average wild chimpanzee.

Domesticated, registered, purebred animals are very-may we say? - gifted specimens of their general species. They are in many instances specially trained, but no training is necessary for many capacities, because a large percentage of the traits are inborn. Most horses cannot be taught to pace, for example, because it involves a form of locomotion which a horse does not naturally utilize. But registered Standardbred foals will start pacing at one or two days of age. Therefore, domesticated animals are not typical of their species, and if this specific point is relevant to their moral standing in any particular way, it probably speaks in their favor, rather than against them.

One might be inclined to note that the traits which these animals have are, of course, traits specifically desired by humans, but again, one could move with that assertion in either direction. Surely the fact that a German short-haired pointer puppy may begin spontaneously to point at an early age does not count against the value of pointers as such, since pointing is merely an exaggeration of traits which most dogs possess. One hesitates to make this assertion flatfootedly, since the counterargument that naturally occurring, untouched species are members of the original primeval environment ahd hence deserve special consideration is strong, but it does not make sense to think that the special qualities of domesticated animals make them somehow less worth preserving that the moa, the Great Auk, or the Indonesian subspecies of rhinoceros. In this sense, I think Rachels' general point serves us well.

Finally, there seems to be a relationship between these special characteristics possessed by many breeds of domesticated animais and the aforementioned hoary topic of sentiency, although the relationship may be somewhat difficult to articulate. Some breeds, particularly of dogs and horses, seem simply to be more sensitive to physical stress and pain than other breeds, and certainly than many non-domesticated animals. Skin characteristics, shape and length of coat, sensitivity to physical stimuli, and so forth, may have become so altered in the course of refining the breed from the original stock that it makes sense to say, in rough terms, that the breed possesses more than average sensitivity or sentiency. And one can certainly say-although the cynical may see this as a topic for humor-that some breeds seem to possess an emotional sensitivity as well. Stereotypes about small dogs and Thoroughbred and Arabian horses are widespread. Again, the inference to be made from this information is that, if these differences between domesticated breeds or strains and non-domesticated types of animals are noted, it would seem that these differences count in their favor, not against them.

In the previous paragraphs I have alluded to the occurrence of non-domesticated species in the pristine, pre-human-intervention environment. This is a topic of some importance and deserves a fuller treatment than I have so far given it. A number of the commentators on the general subject of animal rights seem to feel that arguments for moral consideration of animals break down, crudely, into three categories. Regan, in his article "Ethical Vegetarianism and Commercial Animal Farming," distinguishes between views which we might roughly label utilitarian, deontological, and holis// environmentalist. ${ }^{10}$ Viewed cursorily, the utilitarian position frequently asks us to take animals into consideration morally because they are sentient, or because mistreatment of animals might lead to mistreatment of humans, so that it is human life which 
is primarily valued here. A more straightforward deontological approach grants animals rights, but then the difficulty, as McCloskey has argued, revolves around demarcating the line between right-holding living things and non-right-holding living things and adjudicating disputes, as it were, between animals themselves. ${ }^{11}$ Finally, one can give up on the debate between the consequentialists and the deontologists and simply make the argument that our environment, taken as a whole, is worth preserving. Now, when one does this, one runs into the sorts of conundra which have been parodied by some- the legal standing of trees, and so forth. But more salient for our purposes, one is back to square one in the contretemps over special standing of domesticated animals, because domesticated animals are paradigm cases of living entities which did not naturally occur as part of the environment.

Unfortunately for the case of domesticated animals, it probably can be asserted that if one's interest in animals stems largely or entirely from an interest in the environment as a whole - that is, if one is not much more concemed about the California condor than about the giant sequoia or even the Grand Canyon-one will have comparatively little or no interest in domesticated animals. Those preservationally-minded philosophers, like Passmore and Rodman, ${ }^{12}$ who have written on the need for new attitudes regarding the Earth and the living beings on it have either not dealt with domesticated species at all or have written about animals in a way that indicates that domesticated species might be given short shrift. Passmore, for example, begins his article "Preservation" with the following passage:

By 'preservation' I mean the attempt to maintain in their present condition such areas of the earth's surface as do not yet bear the obvious marks of man's handiwork and to protect from the risk of extinction those species of living beings which man has not yet destroyed. ${ }^{13}$

Passmore goes on to write of the need for preservation due to the intrinsic need of humans for solitude, the various sorts of interpretations of the notion of preservation which might be given by, say, casual campers and vacationers vs. Sierra Club backpackers, and the varying criteria for the usefulness of animal species in general. Toward the end of his piece Passmore discusses in some detail animal rights arguments, but only insofar as they concern non- domesticated animals, and, interestingly enough, he does not think that animal rights arguments themselves are very helpful to the preservationist. Passmore ends his article by writing of a "shift in sensibility," and he continues to write of the need for valuing the environment as a whole. ${ }^{14}$ John Rodman, in "The Liberation of Nature," takes a preservationist stance even more foreign to animal rights than Passmore's. Rodman seems to think that the animal rights arguments are positively harmful, because they send a "double message." It is the second half of the double message, so to speak, which particularly concems Rodman; he writes that "...non-humans are by the same process degraded to the status of inferior human beings, speciesanomalies: imbeciles, the senile, 'human vegetables'-moral half-breeds having rights without obligations (Singer), 'legal incompetents' needing humans to interpret and represent their interests in a perpetual guardian/ward relationship (Stone)."15 In general, Rodman is against the notion of animal rights and argues strenuously for the development of a new attitude toward the natural world. He is also, in general, against the enterprise of domestication itself.

From the foregoing it is possible to glean the following: concern for domesticated species or breeds will have to come from within the animal rights movement. It does not appear to have a natural home within the thought of those who take the holist/environmentalist position with regard to humans and the environment. Problematically, even within the animal rights movement, it seems difficult to separate a concern for domesticated species from a concern for humans. As noted earlier, Regan's work lends itself most naturally to a concern for domesticated species, since so much of his material focuses on commercial animal farming. But the difficulty here is that we are not, presumably, addressing the same issue. I may wholeheartedly agree that the treatment of the Rhode Island Reds used for egg-laying and meat consumption purposes is inhumane, and I may even become a vegetarian. But this is not the same thing as evincing a concern for the preservation of the Rhode Island Red as a breed, in comparison, say, to the Leghorn, or even, again, to the original, non-domesticated Southeast Asia fowl from which our domesticated fowl sprang. I may be against the consumption of horsemeat in France, the use of horsemeat for commercial animal-food purposes here in the U.S., against the preparation of American Saddlebreds for certain shows by using bellboots, and even 
against two-year racing of Thoroughbreds, but none of these of those positions amounts to a concem over the preservation of the American Saddlebred breed as such.

Thus, I think it is clear that within the framework of what has been done so far by philosophers on animal rights, only the position which accords animals actual rights in the sense that humans have rights (this is the position McCloskey, for one, attempts to refute) is at all helpful in the delineation of the status of domesticated species vs. non-domesticated species. Insofar as that position may be countered-and some of the arguments against it are fairly strong ${ }^{16}$-there is a difficulty for both domesticated and non-domesticated species, for any account of the rights of domesticated species or breeds would have to rest on their resemblance to non-domesticated species, as I argued at an earlier point. Insofar as there might be an argument for animal rights which would make characteristics not possessed by domesticated animals salient (for example, capacity to survive genetically without human intervention), it would not be able to encompass rights for domesticated animals. Happily, however, I know of no views of animal rights which are so stringently drawn that they would leave out domesticated animals. Indeed, some views, like Rachels' "moral individualism," which I mentioned earlier, do not take into account group status at all, and this would have the felicitous consequence for domesticated animals of rendering differences between them and non-domesticated species irrelevant from the moral point of view.

In sum I have argued that if we can accept a view of animal rights for non-domesticated species, there are probably no strong reasons for denying the rights to domesticated species, both when seen from the standpoint of the individual creatures involved and when seen from the (more interesting, I believe) standpoint of breeds vs. species. Nevertheless, there remains in the literature a strong strain that what is most valuable about the planet and what is most worth preserving is what is least touched by humans. The inevitable upshot of the ubiquitousness of this view is that many who would be concerned about the Indonesian thinoceros will not be nearly as concerned about the Shire. But the question remains, of course, whether we are concerned about ourselves. The planet as a whole might very well be better off without humans, were there some way to remove humans from the planet without destroying the environment. But we are humans, not beings from another world, and we value human culture. Then, perhaps the strongest counterargument to those who insist on the holist/environmentalist view to the detriment of domesticated animals is that domesticated animals are part of our world, the human world, and by extension, worth preserving.

\section{Notes}

1 John Passmore, "Preservation," in Today's Moral Problems, Richard Wasserstrom, ed. (New York: Macmillan, 1985), passin.

${ }^{2}$ Ibid.

3 Any horse publication provides more than adequate evidence for this assertion. See, in particular, the advertisements in Equus.

${ }^{4}$ lbid.

5 David Hollis, "Morgan Horses Return Home," in Equus, No. 130 (August 1988), pp. 26-29. Around the turn of the century the Morgan nearly vanished as a breed and was only saved by a concerted push.

${ }^{6}$ Peter Singer, "Animal Liberation," in Moral Problems, James Rachels, ed. (New York: Harper \& Row, Inc., 1979) p. 89.

7 James Rachels, "Darwin, Species and Morality," in The Monist, Vol. 70, No. 1 (January, 1987), p. 106.

${ }^{8}$ As research continues to be conducted with the great apes as subjects, this rationalization looks less and less promising.

${ }^{9}$ Rachels, p. 108.

10 Tom Regan, "Ethical Vegetarianism and Commercial Animal Farming," in Wasserstrom.

${ }^{11}$ H. J. McCloskey, "Moral Rights and Animals," in Wasserstrom.

12 John Rodman, "The Liberation of Nature," in Wasserstrom.

13 Passmore, p. 505.

${ }^{14}$ Ibid, p. 527.

15 Rodman, p. 547. Apparently, a similar view informs Bryan G. Norton's Why Preserve Natural Variety? (Princeton, NJ: Princeton University Press, 1988).

${ }^{16}$ See, for example, Paul W. Taylor, "Inherent Value and Moral Rights," for an analysis of technical flaws in Regan's arguments, which Taylor believes vitiate the general animal rights view. The piece appears in The Monist, op. cit. 